\title{
DETERMINATION OF PARTITION COEFFICIENTS AND AQUEOUS SOLUBILITIES BY REVERSE PHASE CHROMATOGRAPHY-I
}

\author{
THEORY AND BACKGROUND
}

\author{
Walter J. Weber JR*, Yu-Ping Chin $\dagger$ and Clifford P. Rice $\ddagger$ \\ Water Resources and Environmental Engineering Program, 181 Engineering Building 1-A, The University \\ of Michigan, Ann Arbor, MI 48109, U.S.A.
}

(Received January 1986)

\begin{abstract}
Water solubilities and octanol/water partition coefficients are widely used to predict partitioning and bioconcentration phenomena for hydrophobic organic pollutants in aqueous systems. This paper is the first in a two part series describing the application of high performance reverse phase liquid chromatography (HPRPLC) for indirect estimation of these two physicochemical parameters to facilitate environmental fate and transport predictions for organic compounds. In the first part, thermodynamic factors which control partitioning processes, water solubilities, and reverse phase retention behavior are discussed, and models for interlinking these three properties are summarized. The second part presents the results of aqueous solubility and octanol/water partition coefficient predictions for a number of organic contaminants from measurements of their HPRPLC behavior, and compares the modeling capabilities of some of the theoretical partitioning/solubility equations developed in the first paper.
\end{abstract}

Key words-organic pollutants, soil/sediment sorption, bioconcentration, activity coefficient, solubility, octanol/water partition coefficient, high performance reverse phase liquid chromatography

\section{NOMENCLATURE}

Roman symbols

$A$ and $C=$ regression coefficients correlating retention times and octanol/water partition coefficients

$a$ and $b=$ regression coefficients correlating partition coefficients for different organic solvent/water systems

$a_{i}^{x} ; a_{i}^{y}=$ solute activity in phases $x$ and $y$

$b(T)$ and $C(T)=$ regression coefficients correlating the free energy of transfer to the solute cavity surface area

$b^{\prime}=$ coefficient correlating the free energy of transfer to retention time

$C_{i}=$ molar concentration $\left(\mathrm{moll}^{-1}\right)$

$\mathrm{CSA}=$ solute cavity surface area

$\Delta C_{p}=$ change in solute heat capacity during phase transition

$d=$ regression coefficient correlating organic solvent/water and hypothetical pure water retention times

$\Delta E=$ cohesive energy

$\Delta E_{m}=$ cohesive energy of mixing

$f_{s}=$ fugacity of crystalline organic solid

$f_{1}^{0}=$ pure liquid component standard state fugacity

\footnotetext{
*Professor of Environmental Engineering and Chairman of the University Water Resources Program.

tGraduate Research Assistant in the Water Resources
} Program.

‡Research Associate in the Great Lakes Research Division.
$\Delta H_{m}=$ enthalpy of mixing

$\Delta H_{f}=$ enthalpy of fusion

$\Delta H_{v}=$ enthalpy of vaporization

$K_{o w}=$ octanol/water partition coefficient

$K_{p}=$ partition coefficient

$K_{R P}=$ reverse phase partition coefficient

$k^{\prime}=$ capacity factor

$\mathrm{MW}=$ molecular weight

$n_{i}=$ moles of solute

$p=$ organic modifier proportionality constant

$\boldsymbol{R}=$ universal gas constant

$R_{M}=$ thin layer chromatography retention factor

$S_{i}=$ molar solubility

$\Delta S_{f}=$ entropy of fusion

$T=$ temperature $\left({ }^{\circ} \mathrm{K}\right)$

$T_{m}=$ solute melting point temperature

$t_{r}=$ solute retention time

$t_{0}=$ mobile phase retention time

$t_{c}=$ corrected retention time

$t_{c}^{w}=$ hypothetical pure water corrected retention time

$t_{c}^{w / 0 r g}=$ corrected retention time in a binary water/organic solvent mobile phase

$V=$ volume

$\nabla=$ molar volume

$\nabla_{i}=$ solute molar volume

$\nabla_{a}^{\prime}=$ octanol molar volume

$\nabla_{\text {ow }}=$ water saturated octanol molar volume

$V_{s}=$ stationary phase volume

$V_{t}=$ total volume of mobile phase required to elute a solute

$V_{v}=$ void space volume

$X_{i}^{x} ; X_{i}^{y}=$ solute mole fraction in $x$ and $y$

$X_{i}^{w}=$ mole fraction solubility in water. 
Greek symbols

$\alpha$ and $\beta=$ regression constants which correlate activity coefficient to retention time

$i_{1}=$ activity coefficient

,$"=$ saturation limit solute activity coefficient in water

" = solute activity coefficient in octanol

= solute activity coefficient in octanol saturated with water

$\delta=$ solubility parameter

$i_{i}=$ solubility parameter of octanol

$\delta_{i j}=$ solubility parameter of octanol saturated with water

$\mu_{1}=$ chemical potential

$\mu_{i}^{\prime \prime}=$ standard state chemical potential

$\Delta \mu_{\text {एrass }}=$ partial molar free energy of transfer

v) = density

\section{INTRODUCTION}

The increased presence of anthropogenic organic pollutants in surface and subsurface waters raises questions concerning the environmental transport and distribution of these materials and their effects on the aquatic community and human health. The manner in which a compound moves and is distributed within an aquatic system and its associated solid phases, both inert (soils, sediments, suspended solids) and living (flora and fauna), is influenced significantly by the physicochemical properties of that compound. Many organic contaminants are "hydrophobic", and tend to sorb from the aqueous phase onto or into phases which are thermodynamically more favorable. The $n$-octanol/water partition coefficient $\left(K_{o w}\right)$ and water solubility $\left(S_{i}^{w}\right)$ are two physicochemical parameters commonly used to predict soil/sediment sorption (Karickhoff et al., 1979, 1984; Briggs, 1973; Means et al.. 1980; Chiou et al., 1982; Voice et al., 1983) and biosorption or bioconcentration phenomena (MacKay, 1982; Chiou et al., 1977; Neely et al., 1974) for lipophilic organic contaminants. Values for these two "predictor" parameters can be determined either experimentally (McAuliffe, 1966; Karickhoff and Brown, 1979; Sutton and Calder, 1974; Haque and Schmedding, 1975; Chiou et al., 1982) or estimated from empirical models (Rekker, 1977; Fujita $e t$ al., 1964; Leo and Hansch, 1979; Arbuckle, 1983). Direct experimental determinations of $n$-octanol/ water partition coefficients are laborious, however, and frequently suffer from lack of precision and accuracy. This is particularly true for very hydrophobic compounds $\left(S_{i}^{w}<50 \mathrm{ppb}\right.$ and $\left.\log K_{o w}>6\right)$. Conversely, estimates of water solubilities and n-octanol/water partition coefficients using such empirical models as are available do not account quantitatively for steric effects and other considerations relating to molecular configuration.

A number of investigators (Veith et al. 1979; McCall, 1975; Unger et al., 1978; Eadsforth and Moser, 1983; Renberg et al., 1980; McDuffie, 1980; D'Amboise and Hanai, 1982; Rapaport and Eisenreich, 1984) have used high performance reverse phase liquid chromatography (HPRPLC) to indirectly estimate $n$-octanol/water partition coefficients. Major advantages of this approach include ease of analysis, speed, precision, and accuracy. Several workers (Locke, 1974; Hakfenschied and Tomlinson, 1981) have also explored the possibility of indirectly estimating water solubility values using HPRPLC. The bulk of this work to date, however, has treated predictions of $S_{i}^{*}$ and $K_{i w}$ from HPRPLC measurements in terms of purely empirical correlations, thus providing no insight to processes which may control relationships between reverse phase chromatographic behavior and solubility and partitioning behavior. The first paper in this two part series summarizes the current level of understanding of concepts underlying solubility and partitioning relationships, and discusses the relevance of these concepts to the selectivity and retention of solutes in reverse phase chromatography systems. Part I begins by examining the relationships of both the phase partitioning behavior and solubility characteristics of a compound to its activity coefficient(s) within a given system. Methods for estimating activity coefficients are then discussed, along with their inherent limitations and inaccuracies. Having established quantitative relationships between the activity coefficient of an organic contaminant and its partitioning and solubility characteristics and associated parameters, Part I then considers diagonal relationships between solubility and partitioning behavior and their related parameters. Finally, the technique of HPRPLC is discussed, its characteristic parameters identified, and these parameters then related and correlated to the characteristic properties of solubility and partitioning. Emphasis is given to the potential application of HPRPLC for quantifying the partitioning and activity coefficients of organic pollutants in aqueous media.

\section{PHASE PARTITIONING AND ACTIVITY COEFFICIENTS}

The partition coefficient, $K_{p}$, quantifies the equilibrium partitioning of a liquid or "supercooled" organic solute between two phases

$$
K_{n}=\frac{C_{i}^{x}}{C_{i}^{i}}
$$

where $C_{i}^{x}$ and $C_{i}^{y}$ are the molar concentrations of solute $i$ in phases $x$ and $y$, respectively.

For conditions of equilibrium between two immiscible solvent phases, the chemical activity, $a_{i}$, of solute $i$ is equal in each phase

$$
a_{\vdots}=a_{i}
$$

Chemically activity is quantified by the expression

$$
a_{1}=y_{i} X_{L}
$$

where $\gamma_{i}$ and $X_{i}$ are the activity coefficient and mole fraction of the solute, respectively. The activity coefficient reflects the degree to which a solution 
deviates from ideality. It is not a constant, however, and varies with $X_{i}$ and $a_{i}$. The two standard states commonly employed for defining the activity coefficient are based on (1) the pure liquid or subcooled liquid component and (2) the "hypothetical" standard state extrapolated from the solute in an infiitely dilute solution (Karger et al., 1973). For purpose of this discussion the former standard state conditions will be specified; that is the solute activity in the region near that of pure component $\left(X_{i}=1\right)$ has a constant value of unity and obeys Raoult's law. In a nonideal aqueous solution, $X_{i}$ approaches zero (the region of infinite dilution), in which case the activity coefficient is greater than unity. The "saturation-limit" solute activity coefficient is used to define the aqueous solubility of hydrophobic organic compounds, and this value differs from those at infinite dilution and near the pure component condition (Tsonopoulos and Prausnitz, 1971).

For the condition of equilibrium, equations (2) and (3) combine to yield

$$
\left(\gamma_{i} X_{i}\right)^{x}=\left(\gamma_{i} X_{i}\right)^{y}
$$

If the concentration of a solute is very low in each phase, its respective mole fractions are given by

$$
X_{i}^{x}=\left(C_{i} \bar{V}\right)^{x} ; X_{i}^{y}=\left(C_{i} \bar{V}\right)^{y}
$$

where $\bar{V}$ is the molar volume of the solvent. The above equations can then be combined to yield an expression for the partition coefficient in terms of the solute activity coefficients in each phase

$$
K_{p}=\frac{\left(\gamma_{i} \bar{V}\right)^{y}}{\left(\gamma_{i} \bar{\nabla}\right)^{x}}
$$

(Chiou et al., 1982; Campbell et al., 1983). This expression is rigorously correct only for totally immiscible phases (i.e. when the respective solubility of each phase in the other is insignificant). The solubility of octanol in water $\left(4.5 \times 10^{-3} \mathrm{M}\right)$, however, may significantly alter an organic solute's activity coefficient, and hence its aqueous solubility. Chiou et al. (1982) observed that the presence of octanol dissolved in water enhances the aqueous solubilities of DDT and hexachlorobenzene by 160 and $80 \%$ respectively. The high solubility of water in octanol $(2.6 \mathrm{M})$ can also cause deviations from equation (6) by changing the properties and altering the molar volume of pure octanol $\left(\bar{V}_{o}=157.7 \mathrm{~cm}^{3} \mathrm{~mol}^{-1}\right)$ to that of a binary octanol-water solution $\left(\bar{V}_{o w}=120 \mathrm{~cm}^{3} \mathrm{~mol}^{-1}\right.$ ) (Arbuckle, 1983; Chiou et al. , 1982; Miller et al., 1985). This combined "mutual solubility" effect offers a potential explanation for the differences between experimentally observed octanol/water partition coefficients and those derived from activity coefficients and equation (6).

The partitioning behavior of a hydrophobic organic substance between water and an organic solvent is controlled largely by the thermodynamic behavior of the solute in the aqueous phase (this will be demonstrated in subsequent sections). It is possi- ble to develop relationships between a solute's partition coefficients for different organic/water systems provided that the solute/organic solvent molecular interactions are limited to weak dispersion forces. Collander (1951) observed that good log-linear correlations exist between the $K_{p}$ values of a hydrophobic compound for different organic solvent/water mixtures if all the organic phases contain the same functional groups. Leo and Hansch (1971) expanded upon the work of Collander, and were able to correlate $K_{p}$ values for different organic solvent/water partition coefficients using the relationship

$$
\log \left(K_{o w}\right)=a\left(\log K_{p}\right)+b
$$

where $a$ and $b$ are empirical constants. They cautioned that this correlation does not necessarily apply to all organic solvent/water mixtures. This is particularly true when comparing systems involving solutes which may specifically interact with the organic phase through polar and/or hydrogen bonding to systems in which most of the solute-organic solvent interactions are controlled by physical dispersion forces (Leo and Hansch, 1971; Campbell et al., 1983).

\section{AQUEOUS SOLUBILITY AND ACTIVITY COEFFICIENTS}

The aqueous solubilities of liquid hydrophobic organic compounds (for which the solute melting point temperature, $T_{m}$, is $<25^{\circ} \mathrm{C}$ ) can be approximated directly from their activity coefficients. Further thermodynamic considerations are required to estimate "supercooled" solute activities for substances that are crystalline solids $\left(T_{m}>25^{\circ} \mathrm{C}\right)$. A good first approximation of the liquid solute activity for a sparingly soluble hydrophobic compound is unity, that is, the same as its pure component activity as specified by the standard state (because of the limited solubility of water in the organic compound). Solubility in terms of mole fractions is merely the reciprocal of the "saturation limit" aqueous solute activity coefficient

$$
X_{i}=1 / \gamma_{i}^{w}
$$

and the molar solubility, $S_{i}^{w}$, is simply the product of the mole fraction solubility and the molarity of water $\left(55.5 \mathrm{~mol} \mathrm{l}^{-1}\right.$ ) (Yalkowski and Valvani, 1980). The relationship between $S_{i}^{w}$ and $X_{i}^{w}$ is thus log linear

$$
\log \left(S_{i}^{w}\right)=\log \left(X_{i}^{w}\right)+1.74
$$

Estimation of the aqueous solubility of crystalline organic solids (high $T_{m}$ ) requires a more rigorous thermodynamic treatment. Since the pure liquid was specified as the standard state, the activity of an organic solute is defined as

$$
a_{i}=f_{s} / f_{1}^{0}
$$

where $f_{s}$ and $f_{1}^{0}$ are the fugacities of the crystalline solid and the pure liquid standard state respectively. The solubility of the solid can be determined by estimating its hypothetical "supercooled liquid" 
activity (Hildebrand et al., 1970; Yalkowski and Valvani, 1980; Chiou et al., 1982). The activity of the "supercooled solute" is no longer unity and is estimated from the enthalpy of fusion, $\Delta H_{f}$,

$$
\begin{aligned}
\log \left(a_{i}\right)=\frac{-\Delta H_{f}}{2.3 R} & {\left[\frac{T_{m}-T}{T_{m}(T)}\right] } \\
+ & \frac{\Delta C_{p}}{R}\left[\frac{T_{m}-T}{T}\right]-\frac{\Delta C_{p}}{R} \ln \frac{T_{m}}{T}
\end{aligned}
$$

where $\boldsymbol{R}$ is the universal gas constant, $T$ is the equilibrium temperature $(\mathrm{K})$, and $\Delta C_{p}$ is the change in the solute heat capacity upon melting. The change in heat capacity for many crystalline solid organic compounds is small, and as a first approximation may be considered negligible (Yalkowski and Valvani, 1980). This simplifies equation (11a) to

$$
\log \left(a_{i}\right)=\frac{-\Delta H_{f}}{2.3 R}\left[\frac{T_{m}-T}{T_{m} T}\right]
$$

The mole fraction aqueous solubility of solid organic solutes can be determined from the relationship

$$
\log \left(X_{i}^{w}\right)=\log \left(a_{i}\right)-\log \left(\gamma_{i}^{w}\right)
$$

Combination of equations (11b) and (12) yields

$$
\log \left(X_{i}^{w}\right)=\frac{-\Delta H_{f}}{2.3 \boldsymbol{R}}\left(\frac{T_{m}-T}{T_{m} T}\right)-\log \left(\gamma_{i}^{w}\right) .
$$

Molar aqueous solubility can be estimated by combining equations (9) and (13)

$\log \left(S_{i}^{w}\right)=\frac{-\Delta H_{f}}{2.3 R}\left(\frac{T_{m}-T}{T_{m} T}\right)-\log \left(\gamma_{i}^{*}\right)+1.74$.

It is evident that accurate determination of the aqueous solubility of an organic compound is strongly dependent upon reliable estimates of the saturation limit activity coefficient and the heat of fusion (Yalkowski and Valvani, 1979, 1980). While the change in heat capacity during the solute phase transition may be insignificant for many compounds, the solute heat capacity term should be incorporated if available. Tsonopoulos and Prausnitz (1971) observed that the difference between including and excluding the heat capacity term for 4-chlorobenzoic acid altered its activity coefficient by over a half order of magnitude. Subsequent sections present methods which can be used to estimate heats of fusion and activity coefficients in apolar solvents and water.

\section{ESTIMATING ACTIVITY COEFFICIENTS}

\section{Regular solution theory}

It is apparent from the foregoing analysis that both the solubility and the phase partitioning characteristics of an organic compound can be related to its activity coefficients in the phase of interest. The regular solution theory (RST) provides a reasonably accurate means for predicting activity coefficients for organic solutes in apolar solvents.

Molecular interactions (solute-solute, solvent- solvent, and solute--solvent) in solutions which behave ideally are assumed to be equal. The RST predicts deviations from ideal solution behavior by estimating the changes in system enthalpy which occur upon mixing, $\Delta H_{m}$. A property termed the "cohesive energy density" of a pure substance is used to quantify the enthalpy of mixing term. This property is the energy required to vaporize 1 mole of pure liquid; that is, the energy per unit molar volume $(\Delta E / V)$. The familiar solubility parameter, $\delta$, is given by the square root of the "cohesive energy density"

$$
\vec{\partial}=(\Delta E / \bar{V})^{0.5} \text {. }
$$

The solubility parameter of a substance can be estimated with reasonable accuracy from the substance's heat of vaporization, $\Delta H_{t}$

$$
\delta=\left[\frac{\rho\left(\Delta H_{r}-R T\right)}{\mathrm{MW}}\right]^{0.5}
$$

where $\rho$ is the density of the compound, MW its molecular weight, and all other terms are as previously defined (Hildebrand et al., 1970; Barton. 1975). Values for the heat of vaporization can be found in chemical handbooks for many compounds, or estimated from known vapor pressure values using the Clausius-Clapeyron equation.

The regular solution theory, like many conceptual models, is based upon several assumptions; namely (1) no volume change occurs upon mixing; (2) the entropy of mixing is ideal; and (3) all molecular interactions in solution are attributable to London/Van der Waals forces. The last assumption severely limits the applicability of the model to the dissolution of organic solutes in apolar solvents.

For dissolution of solute $i$ in solvent $s$, the chemical potential of $i, \mu_{i}$, is defined in terms of its standard state partial molal free energy, $\mu_{i}^{*}$, and its activity, $a_{i}$, as

$$
\mu_{i}=\mu_{i}^{\prime}+R T \ln \left(a_{i}\right)
$$

or, from equation (3)

$$
\mu_{i}=\mu_{i}^{\prime \prime}+R T \ln \left(\gamma_{i} X_{i}\right) .
$$

Deviations from ideal behavior in regular solutions are quantified by changes in the enthalpy of a system upon the mixing of $i$ and $s$ to form an infinitely dilute solution (Hildebrand et al., 1970; Karger et al., 1973)

$$
\mu_{i}-\mu_{i}^{\prime \prime}=\Delta H_{m}+\boldsymbol{R} T \ln \left(X_{i}\right)
$$

The combination of equations (18) and (19) yields an expression which approximates the solute activity coefficient

$$
\ln \left(\gamma_{i}\right)=\frac{\Delta H_{m}}{R T}
$$

If it is assumed that no volume change occurs upon mixing, the cohesive energy of mixing, $\Delta E_{m}$, for a liquid solution can be taken as equal to the enthalpy of mixing term

$$
\Delta H_{m}=\Delta E_{m} .
$$


The term, $\Delta E_{m}$, can be approximated from solubility parameters for the solute and solvent using the Hildebrand geometric mean assumption (Hildebrand et al., 1970; Karger et al., 1973)

$$
\Delta E_{m}=\bar{\nabla}_{i}\left(\delta_{i}^{2}-2 \delta_{i} \delta_{s}+\delta_{s}^{2}\right) .
$$

Substitution of equation (21) into (22), and simplification of the polynomial yields

$$
\Delta H_{m}=\bar{V}_{i}\left(\delta_{i}-\delta_{s}\right)^{2}
$$

Introduction of equation (23) into (20), and conversion to a common logarithm base gives

$$
\log \gamma_{i}=\frac{\bar{V}_{i}\left(\delta_{i}-\delta_{s}\right)^{2}}{2.3 R T} \text {. }
$$

If the difference between the solvent and solute solubility parameters is negligible $\left(\delta_{i}=\delta_{s}\right)$, the solution approaches ideality.

The applicability of this method for estimating activity coefficients is restricted by the assumptions presented earlier. For solutions in which specific solute-solvent or strong solvent-solvent interactions are operative, the regular solution model is not adequate for prediction of activity coefficients. Hagen and Flynn (1983) reported that the regular solution model prediction for the activity coefficient of hydrocortisone in water deviated from an experimentally derived value by nearly 21 orders of magnitude. This example clearly illustrates that differences in the molar cohesive energy densities between the solute and solvent cannot adequately account for specific molecular interactions which occur in a solution. Solubility parameters, however, can provide a reasonable first approximation of whether a particular solute and solvent are at all compatible. The RST model can also predict with reasonable accuracy the activity coefficients of nonelectrolytic organic solutes in apolar solvents (Hagen and Flynn, 1983)

\section{The solvophobic theory and solute surface area model}

The solvophobic theory provides a conceptual basis and predictive method for describing the behavior of organic compounds in water or solvents which may participate in hydrogen bonding. The model recognizes that the solubility of nonelectrolytic organic substances is controlled largely by the thermodynamic properties of the solvent. Frank and Evans (1945) first proposed such a concept when they observed large decreases in the entropies of apolar solute/water solutions upon mixing. They attributed this phenomenon to the formation of "iceberg" water structures around each apolar solute molecule. Molecular associations of water can be depicted conceptually as existing in one of two possible structural configurations, depending upon the number of associated hydrogen bonds in which the solvent molecule can participate. Water molecules at interfaces are bound to each other by hydrogen bonds to four other molecules to form "ice-like" polyhedra (Voice and Weber, 1983; Nemethy and Scheraga,
1962). This partially accounts for the high surface tension of water. Water molecules in the bulk phase are more randomly agglomerated, but still interact through relatively strong dipole interactions. The introduction of an apolar compound requires the formation of a cavity in the solvent to accomodate the solute molecule. This brings about the replacement of water-water dipole interactions by favorable, but weaker solute-water interactions affected primarily by London/Van der Waals forces. These physical dispersion interactions decrease sharply with the distance of molecular separation (as the sixth power of distance) and are therefore insignificant beyond the first layer of water molecules. The degree of hydrogen bonding between water molecules, however, increases significantly in the immediate vicinity of a solute molecule, extending it to form a partial cage of ice-like polyhedral structures. This increased ordering of solvent molecules results in a decrease in system entropy, more than offsetting the modest gain of enthalpy from mixing between the solute and solvent.

Nemethy and Scheraga (1962) used partition functions to estimate differences in the free energy of water molecules in bulk phase and those at solvent/solute interfaces. They observed that the activities (and hence solubilities) of hydrocarbons are dependent upon the number of water molecules in the solvent layer adjacent to the solute molecules. Several workers (Hermann, 1971; Amidon et al., 1975; Valvani et al., 1976; Yalkowski and Valvani, 1979) have shown that a good correlation exists between the surface area of the solvent cavity and the solubility of aliphatic and aromatic hydrocarbons. The dissolution of nonelectrolytic organic solutes in water can be conceptualized as involving three processes; namely, (1) dissociation of solute from the pure liquid, (2) the formation of cavities in the solvent to accept solute molecules and (3) insertion of solute into the cavities (Amidon et al., 1975). The free energy of transfer, from the pure solute liquid or supercooled liquid to water, $\Delta \mu_{\text {trans. }}$ is given by

$$
\Delta \mu_{\text {trans. }}=-R T\left(\ln X_{i}^{w}\right) \text {. }
$$

Combining this expression with equation (8) yields an expression for the free energy of transfer as a function of the activity coefficient

$$
\Delta \mu_{\text {trans. }}=R T\left(\ln \gamma_{i}^{w^{w}}\right) .
$$

The free energy of transfer term is linearly correlated to the cavity surface area, CSA, by

$$
\Delta \mu_{\text {trans. }}=b(T) C S A+C(T)
$$

where $b(T)$ and $C(T)$ are temperature dependent coefficients (Hermann, 1971). Substitution of equation (27) into (26) yields an expression for the activity coefficient as a function of the CSA

$$
\log \gamma_{i}^{w}=\left[\frac{b(T) C S A+C(T)}{2.3 R T}\right]
$$


Hermann (1971), Amidon et al. (1975), and Valvani et al. (1976) have used variations of equation (28) successfully to predict the aqueous solubilities of a number of hydrocarbons. Calculations for the CSA values of hydrocarbons are based upon conceptualization of a solute molecule as intersecting spheres of carbon, hydrogen, and oxygen atoms, in which each atom has a fixed radius. A solvent radius of $1.5 \AA$ is added to the radius of each atom, and the surface area computed using the combined solvent-solute radii and taking into account intramoleuclar bond lengths and bond angles for each solute molecule. Thorough treatments of methods for calculating CSA values have been given by Herman (1971), and Valvani et al. (1976).

\section{PARTITION COEFFICIENTS AND SOLUBILITY}

It has been demonstrated in preceding sections that the solubility, $S_{i}^{*}$, and the partition coefficient, $K_{o w}$, of an organic compound are both related to the activity coefficient of that compound in a given phase. It is therefore reasonable to expect a diagonal relationship between $S_{i}^{w}$ and $K_{o n}$. Yalkowski and Valvani (1980) recognized that many organic substances behave almost ideally in octanol $\left(\gamma_{i}^{o}=1\right)$. For an octanol/water partitioning system the relationship given in equation (6) becomes

$$
K_{n w}=\left[\frac{\gamma_{1}^{w} \bar{V}_{n}}{\bar{V}_{n}}\right]
$$

or, in logarithmic form

$$
\log \left(\gamma_{i}^{w}\right)=\log \left(K_{o w}\right)+0.94
$$

where $\log \left(\bar{V}_{o} / \bar{V}_{w}\right)$ is equal to 0.94 . If the "mutual solubility" effect into consideration, the molar volume of the water-saturated octanol phase, $\bar{V}_{o w}$, becomes $120 \mathrm{~cm}^{3} \mathrm{~mol}^{-1}$, the equation (30) becomes

$$
\log \left(\gamma_{i}^{w}\right)=\log \left(K_{\text {ow }}\right)+0.82 \text {. }
$$

Once the activity coefficient for a compound in a particular solvent has been determined, it is also possible to calculated the ideal solubility because the enthalpy of fusion can be approximated by the entropy of fusion, $\Delta S_{f}$, from the relationship

$$
\Delta H_{f}=\Delta S_{f} T_{m}
$$

Combining equation (11b) and (32) yields

$$
\log \left(a_{i}^{w}\right)=\frac{-\Delta S_{f}}{2.3 R T}\left(T_{m}-T\right)
$$

Equations (14), (30) and (32) combine to give an expression which interrelates the aqueous molar solubility of an organic non-electroyte and its octanol/ water coefficients

$$
\log \left(S_{i}^{w}\right)=\frac{\Delta S_{f}}{2.3 R T}\left(T_{m}-T\right)-\log \left(K_{o w}\right)+0.80
$$

This model assumes that "mutual solubility" is negligible (Yalkowski and Valvani, 1980). If one considers the "mutual saturation" of the two partitioning phases, equation (31) is used in lieu of equation (30) to yield

$$
\log \left(S_{i}^{w^{\prime}}\right)=\frac{-\Delta S_{f}}{2.3 R T}\left(T_{M}-T\right)-\log \left(K_{o w}\right)+0.92 .
$$

Equation (34) has been reported to provide good correlation between water solubilities and octanol/ water partition coefficients for polycyclic aromatic compounds, halobenzenes, and alkyl p-substituted benzoates (Yalkowski and Valvani, 1980). The use of equation (35) for predicting the solubilities of aromatic compounds from their respective octanol/water partition coefficients is considered in Part II of this series.

Many non-electrolyte organic compounds deviate appreciably from ideal behavior in octanol. The solubility parameter of octanol is 10.3 , while compounds such as mesitylene, o-xylene, and trichlorobenzene, to name a few, have solubility parameters that are $<9.3$. The RST model can be applied to predict activity coefficients for organic solutes under such circumstances by first considering the logarithmic form of equation (6) for an octanol/water partitioning system (in which $K_{p}=K_{o w}$ ) with mutual saturation

$$
\log \left(\gamma_{i}^{w}\right)=\log \left(K_{p w}\right)+\log \left(\gamma_{i}^{\prime w}\right)+0.82
$$

where $\gamma^{o w}$ is the activity coefficient of the binary water saturated octanol phase and $\log \left(\bar{V}_{o w} / \bar{V}_{w}\right)$ is equal to 0.82. Equation (24) (RST) can be used to predict $\log \left(\gamma_{i}^{o w}\right)$, and its subsequent substitution into equation (36) yields

$$
\log \left(\gamma_{i}^{w}\right)=\log \left(K_{o w}\right)+\frac{\bar{V}_{i}\left(\delta_{i}-\delta_{o}^{\prime}\right)^{2}}{2.3 R T}+0.82
$$

where $\delta_{o}^{\prime}$ is the solubility parameter of the binary water/octanol organic phase.

Combination of equations (14), (32) and (37) results in the expression

$$
\begin{array}{r}
\log \left(S_{i}^{n}\right)=\frac{\left[-\Delta S_{f}\left(T_{m}-T\right)-\bar{V}_{i}\left(\delta_{i}-\delta_{o}^{\prime}\right)^{2}\right]}{2.3 \boldsymbol{R} T} \\
-\log \left(K_{o w}\right)+0.92 .
\end{array}
$$

If the "mutual solubility" effect is not taken into account, equation (38) becomes

$$
\begin{array}{r}
\log \left(S_{i}^{k}\right)=\frac{\left[-\Delta S_{f}\left(T_{m}-T\right)-\bar{V}_{i}\left(\delta_{i}-\delta_{o}\right)^{2}\right]}{2.3 R T} \\
-\log \left(K_{o w}\right)+0.80
\end{array}
$$

where $\delta_{0}$ is the solubility parameter of pure octanol. This results in a model quite similiar to the one developed by Amidon and Williams (1982). The predictive capabilities of equation (38) will also be considered in Part II.

Thus, by virtue of their mutual dependence on the activity coefficient, the solubility, octanol/water partitioning, and distribution coefficients of an organic compound can be interrelated. It is of interest now to 
explore the experimental determination of distribution coefficients by HPRPLC and the estimation of solubility and octanol/water partition coefficients from such measurements.

\section{PARTITION CHROMATOGRAPHY AND DISTRIBUTION COEFFICIENTS}

Reverse phase chromatography has become a popular analytical methodology since the relatively recent advent and refinement of instrumentation for high performance liquid chromatography. A reverse phase packings is comprised of a nonpolar stationary phase which is either mechanically held or chemically bonded to an inert support medium. For measurements of partitioning from aqueous solutions the mobile phase is composed of a fixed ratio (isocratic) of water and organic solvent (methanol or acetonitrile). The exact ratio of water to organic modifier and the type of organic solvent used are dependent upon the physicochemical properties of the target compounds.

Most modern reverse phase packings are comprised of $n$-octyldecyl groups chemically bonded to a silica support medium. Several investigators (Horvath et al, 1976; Colin and Guichon, 1977; Karger et al., 1976) have developed models to describe the sorptive processes which occur within a bonded reverse phase column. It has been speculated that solvophobic interactions are primarily responsible for driving solute molecules out of solution and onto the stationary phase. This is no clear consensus, however, on whether the process is strictly surface adsorption or phase partitioning. Horvath and co-workers (1976) have attributed the separation to a combination of partitioning and adsorption. The stationary phase can be envisioned as being covered by a "molecular fur" of alkyl chains. If the surface of the support were covered by a monolayer of these alkanes the distances between each hydrocarbon molecule would be relatively large, and it is improbable that such a stationary phase would behave like a true liquid. The alkyl groups would have fewer translational and rotational degrees of freedom, which would inhibit solute migration within the stationary phase, and this would make true partitioning an unlikely process. Conversely, separation by true adsorption might also be excluded on the basis that the association of the solute with the bonded phase seems to be solvophobic in nature. Irrespective of the precise mechanism involved, however, the retention time of the compound is directly related to the degree of partitioning that occurs.

The degree of partitioning in reverse phase chromatography is quantified in terms of a solute distribution coefficient, $K_{R P}$, characterizing the equilibrium ratio of solute concentration in the stationary and mobile phase i.e. $K_{R P}=C_{f}^{s} / C_{i}^{m}$. This constant is analogous to the partition coefficient defined in equation (1). The volume of a solvent required to elute a solute associated with the stationary phase in a HPRPLC column, $V_{t}$, is related to $K_{R P}$; the larger the value of $K_{R P}$, the larger the value $V_{t}$ of the mobile phase required to elute the solute. Indeed, this volume, corrected for the volume, $V_{v}$, retained in the pore spaces of the column, is directly equal to $K_{R P}$ times the volume of the solid phase, $V_{s}$; that is

$$
V_{1}-V_{v}=K_{R P}\left(V_{s}\right)
$$

(Karger et al., 1973). The most common way of expressing the retention capacity of a column is through the use of a capacity factor, $k^{\prime}$, which is the equilibrium ratio of the number of moles of solute in the stationary and mobile phases i.e. $n_{i}^{s} / n_{i}^{m}$.

Thus,

$$
K_{R P}=\frac{C_{i}^{s}}{C_{i}^{m}}=\frac{n_{i}^{s}}{n_{i}^{m}}\left[\frac{V_{v}}{V_{s}}\right]=k^{\prime}\left[\frac{V_{v}}{V_{s}}\right] .
$$

The combination of equations (40) and (41) yields an expression for the capacity factor as a function of $V_{t}$ and $V_{v}$

$$
k^{\prime}=\frac{V_{t}-V_{v}}{V_{v}} .
$$

The capacity factor thus quantifies the distribution behavior of an organic compound in a reverse phase column. This variable can be related in turn to the solute retention time, $t_{r}$, by dividing each of the volume terms in equation (42) by the flowrate of the mobile phase to give

$$
k^{\prime}=\frac{t_{r}-t_{0}}{t_{0}}
$$

where $t_{0}$ is the retention time of the solvent. It is theoretically possible, therefore, to determine values for the capacity factor from measurements of $t_{r}$ and $t_{0}$ for a particular solute/solvent combination. However, Krstulovic et al. (1982) have found that different "unretained" tracers $\left({ }^{14} \mathrm{C}\right.$-methanol, acetone, uracil, sodium nitrate) commonly used to determine $t_{0}$ yield inconsistent results. Different mobile phase compositions have been found to yield different solvent retention times. Nonetheless, because $k^{\prime}$ is directly proportional to $t_{r}$ and $K_{R P}$, it is possible, for purposes of quantifying partitioning processes in reverse phase columns, to employ a "corrected retention time", $t_{c}$, (the solute retention time minus the retention time of a relatively unretained internal standard) in lieu of the capacity factor. It can be assumed that any environmental changes (i.e. temperature, flowrate, etc.) will affect the solute and internal standard in the same manner. Again, because of the direct proportionality relationships, $t_{c}$ can be used in lieu of $K_{R P}$ to correlate HPRPLC results with the octanol/water partition coefficients in equation (7). Thus

$$
\log \left(K_{o w}\right)=A\left(\log t_{c}\right)+C .
$$

The constants $A$ and $C$ are determined empirically by calibrating the HPRPLC instrument to a group of organic substances which have well-defined 
octanol/water partition coefficients, under constant temperature, flowrate, pressure, and mobile phase concentrations. Target compounds are then analyzed under identical conditions and their octanol/water partition coefficients are estimated from their retention times using an appropriately calibrated form of equation (44).

\section{PARTITION CHROMATOGRAPHY AND SOLUBILITY}

The preceding section outlines the conceptual basis for applying HPRPLC measurements to estimation of octanol/water partition coefficients for organic compounds. Octanol/water partition coefficients and solubility are both related to the activity coefficient and therefore to each other, as developed earlier in this paper. It is thus reasonable to assume that the activity coefficient and solubility of a compound can also be related to its HPRPLC retention time. Locke (1974) observed that the elution order of hydrophobic substances from a reverse phase column can be correlated to solute solubility. This correlation is based on the assumptions that solvophobic interactions are the primary thermodynamic driving forces, that no stable complexes are formed, that the solute/stationary phase interactions are weak and nonspecific, that the stationary phase surface is relatively homogeneous, and that the surface activity coefficients of all the eluting solutes are similar.

It is possible by applying the assumptions described above to derive a thermodynamic model in which the selectivity and retention of an organic compound are strictly dependent upon its solvation in a polar solvent. If water is used as the mobile phase, then, for a given solute, the free energy of transfer term defined previously can be linearly correlated to the logarithm of the corrected retention time, $t_{c}^{n}$

$$
\log \left(t_{c}^{w}\right)=\left[\frac{\Delta \mu_{\text {trans. }}}{2.3 R T}\right]+b^{\prime}
$$

where $b^{\prime}$ is a constant (Hakfenschied and Tomlinson, 1981). Substitution of equation (26) into equation (45) after conversion to base 10 logarithmic form yields

$$
\log \left(t_{c}^{w}\right)=\log \left(\gamma_{i}^{w}\right)+b^{\prime}
$$

Because it is not feasible to elute hydrophobic substances with pure water in reverse phase chromatography, it is necessary to develop a correlation between the hypothetical water eluent $t_{c}^{w}$ value and that of a water/organic modifier eluent, $t_{c}^{w / o r g}$. Soczewinski and Wachtmeister (1962) determined theoretically and verified experimentally that the logarithm of retention factor, $R_{m}$, for a solute in paper chromatography changed linearly with the amount of organic modifier present in the mobile phase. Several subsequent workers have shown that $\log$ retention factors in reverse phase thin layer chromatography (RPTLC) and HPRPLC retention times may vary linearly with the amount of organic solvent in the mobile phase (Biagi et al., 1979; Butte et al., 1981; Kringstad et al., 1984). The hypothetical pure water corrected retention time, $t^{w}$, for a particular organic solute can be determined from the relationship

$$
\log \left(t_{c}^{w}\right)=\log \left(t_{c}^{\text {worg }}\right)-p(\% \text { organic modifier })
$$

where $p$ is a proportionality constant. Determining the hypothetical pure water corrected retention time for each solute would be a laborious process; a more direct correlation between $t_{c}^{\text {wiorg }}$ for a particular isocratic water/organic solvent mobile phase and the solute's activity coefficient in water is therefore desirable. Because retention times are simply reflections of sorptive processes in HPRPLC, $\log \left(t_{c}^{w}\right)$ can be correlated to the $\log \left(t^{*}\right.$ org $)$ by a relationship of the form given in equation (7), i.e.

$$
\log \left(t_{c}^{*}\right)=\alpha\left[\log \left(t_{c}^{\text {w.org }}\right)\right]+d
$$

where $\alpha$ and $d$ are empirical coefficients. Using the data of Biagi et al. (1979), the hypothetical pure water retention factors, $R_{m}^{*}$, and experimentally derived acetone/water $(40: 60)$ eluted retention factors, $R_{m}^{w / a c e t}$, for the separation of napthol and its derivatives using a silicone oil RPTLC system were calculated; these are presented in Table 1 and plotted in Fig. 1 (retention factors are the RPTLC equivalent to the logarithm of HPRPLC capacity factors). The linearity between the two parameters is excellent (correlation coefficient $=0.99$ ), in accord with the relationship given in equation (48). Substitution of equation (48) into equation (46) yields

$$
\log \left(\gamma_{i}^{n}\right)=\alpha \log \left(t_{c}^{n} \text { urg }\right)+\beta
$$

where $\beta$ is equal to $\left(d-b^{\prime}\right)$. Equation (49) allows one to directly correlate the aqueous activity coefficients of organic compounds to their water/organic solvent eluted HPRPLC $t_{c}$ values. This simplifies things greatly by circumventing the extrapolation of $\log \left(t_{i}^{w}\right)$.

The determination of aqueous solubilities is done by combining equations (49), (32) and (14) to yield

$$
\begin{aligned}
& \log \left(S_{i}^{w}\right)=1.74-\frac{\Delta S_{f}\left(T_{m}-T\right)}{2.3 R T} \\
&-x \log \left(t_{i}^{\text {worg }}\right)-\beta .
\end{aligned}
$$

The constants $\alpha$ and $\beta$ must be determined empir-

Table 1. Hypothetical pure water and experimental acetone/water $(40 / 60)$ reverse phase thin layer chromatography retention factors for napthol and its derivatives

\begin{tabular}{lcc}
\hline Compound & $R_{m}^{w / a c t *}$ & $R_{m}^{*}$ \\
\hline 1, 2, 3, 4-tetrahydrol- & 0.28 & 0.75 \\
1-napthol & & \\
2-methyl-1-napthol & 0.03 & 1.67 \\
1-bromo-2-napthol & 0.08 & 1.86 \\
4-chloro-1-napthol & 0.24 & 2.08 \\
2-napthol & 0.14 & 1.28 \\
1, 6-dibromo-2-napthol & 0.42 & 2.50 \\
5, 6, 7, 8-tetrahydro-1-napthol & 0.01 & 1.52 \\
6-bromo-2-napthol & 0.21 & 2.08 \\
\hline
\end{tabular}

${ }^{*} R_{m}^{\text {w/ace }}=\log \left(1 / R_{f}-1\right)$.

From Biagi et al. (1979) 


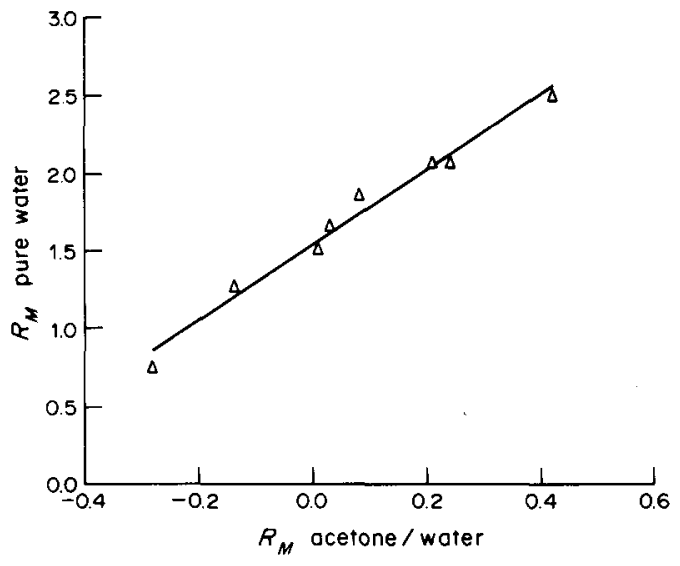

Fig. 1. Correlation between experimentally determined acetone/water and hypothetical pure water reverse phase thin layer chromatography retention factors for napthol and its derivatives.

ically by calibrating the HPRPLC instrument to the aqueous activity coefficients of several organic compounds under constant temperature, flowrate, and mobile phase composition. The retention times for a target compound are then determined under identical conditions. Caution should be exercised regarding selection of the organic solutes used for calibration, because several classes of organics (particularly the pyridines and analine) can participate in specific interactions with the stationary phase.

Estimates of aqueous solubility using HPRPLC measurements in conjunction with equation (50) should be as good as or superior to those made from octanol/water partition coefficients, particularly for very hydrophobic compounds. The use of reverse phase chromatography eliminates errors arising from mutual saturation effects because the solubility of the $\mathrm{C}_{18}$ stationary phase is insignificant in the mobile phase. The accuracy and precision of the technique are further enhanced by the use of an internal standard.

\section{CONCLUSIONS}

A number of efforts have been made to empirically correlate certain environmental fate and transport processes for organic pollutants (e.g. bioconcentration and soil/sediment sorption) to surrogate "predictor" values (e.g. $n$-octanol/water coefficient and water solubilities), that characterize the physicochemical properties of those pollutants. Estimates of such "predictors" can be based in turn on measurements of high performance reverse phase liquid chromatography capacity factors and retention times. Analysis of the thermodynamic models used to interlink HPRPLC capacity factors, water solubilities, and partition coefficients result in several conclusions: (1) the partition coefficient, water solubility, and capacity factor for a solute can all be correlated to its saturation-limit aqueous activity coefficient; (2) organic solutes may behave like regular solutions in apolar solvents; (3) organic solutes are strongly influenced by solvophobic interactions in water; (4) HPRPLC retention times can be used to quantify the distribution behavior of a solute in reverse phase systems; (5) the distribution coefficients of a solute for partitioning from water into different organic solvent systems can be correlated to each other, provided that no specific molecular interactions occur between the solute and the organic solvents; and, (6) the retention and selectivity of a solute in a reverse phase system appears to be dependent upon its mobile phase activity coefficient.

Acknowledgements - The authors thank Dr Norman Wiener and Dr Gordon Flynn of the University of Michigan College of Pharmacy for their helpful comments. The work presented in this two part series has been supported in part by the Michigan Sea Grant Program, and in part by the Cooperative Program between the Great Lakes Environmental Research Laboratory of the National Oceanic and Atmospheric Administration and the University of Michigan.

\section{REFERENCES}

Amidon G. L. and Williams N. A. (1982) A solubility equation for nonelectrolytes. Int. J. Pharm. 11, 249-256.

Amidon G. L., Yalkowski S. H., Anik S. T. and Valvani S. C. (1975) Solubility of nonelectrolytes in polar solventsV. Estimation of the solubility of aliphatic monofunctional compounds in water using a molecular surface area approach. J. phys. Chem. 79, 2239-2245.

Arbuckle W. B. (1983) Estimating activity coefficients for use in calculating environmental parameters. Envir. Sci. Technol. 17, 537-542.

Barton A. F. M. (1975) Solubility parameters. Chem. Rev. 75, 731-751.

Biagi G. L., Barbaro A. M., Guerra M. C., Hakim G. and Borea P. A. (1979) $R_{m}$ values of napthols and acetophenones in structure activity studies. J. Chromat. 177, 35-49.

Briggs G. G. (1973) A simple relationship between soil adsorption of organic chemicals and their octanol/water partition coefficients. Proc. 7th British Insecticide and Fungicide Conf. 11, 475-478.

Butte W., Fooken C., Klussman R. and Schuller D. (1981) Evaluation of lipophilic properties for a series of phenols using reverse phase high performance liquid chromatography and high performance thin layer chromatography. J. Chromat. 214, 59-67.

Campbell J. R., Luthy R. G. and Carrondo M. J. T. (1983) Measurement and prediction of distribution coefficients for wastewater aromatic solutes. Envir. Sci. Technol. 17, 582-590.

Colin H. and Guichon G. (1977) Introduction to reverse phase high performance liquid chromatography. J. Chromat. 141, 289-312.

Collander R. (1951) The partitioning of organic compounds between higher alcohols and water. Acta chem. scand. 5, 774-780.

Chiou C. T., Schmedding D. W. and Manes M. (1982) Partitioning of organic compounds in octanol-water systems. Envir. Sci. Technol. 16, 4-10.

Chiou C. T., Freed V. H., Schmedding D. W. and Kohnert R. L. (1977) Partition coefficient and bioaccumulation of selected organic chemicals. Envir. Sci. Technol. 11, 475-478.

D'Amboise M. and Hanai T. (1982) Hydrophobicity and 
retention in reverse phase liquid chromatography. $J$. Liq. Chromat. 229-244.

Eadsforth C. V. and Moser P. (1983) Assessment of reverse phase chromatographic methods for determining partition coefficients. Chemosphere, 12, 1459-1475.

Frank H. S. and Evans M. (1945) Free volume and entropy in condensed systems. J. chem. Phys. 13, 507-532.

Fujita T., Iwasa J. and Hansch C. (1964) A new substituent coefficient, $\pi$, derived from partition coefficients. $J$. Am. chem. Soc. 86, 5175-5180.

Hagen T. A. and Flynn G. A. (1983) Solubility of hydrocortisone in organic and aqueous media: evidence for regular solution behavior in apolar solvents. J. Pharm. Sci. $72,409413$.

Hakfenscheid T. L. and Tomlinson E. (1981) Estimation of aqueous solubilities of organic nonelectrolytes using liquid chromatography data. J. Chromat. 218, 409-425.

Haque R. and Schmedding D. W. (1975) A method of measuring the water solubility of hydrophobic chemicals: solubility of five polychlorinated biphenyls. Bull. envir. contam. Toxic. 14, 13-18.

Hermann R. B. (1971) Theory of hydrophobic bonding-II. The correlation of hydrocarbon solubility in water with solvent cavity surface area. J. phys. Chem. 76, 2754-2759.

Hildebrand J. H., Prausnitz J. M. and Scott R. L. (1970) Regular and Related Solutions. Van Nostrand Reinhold, New York

Horvath C.. Melander W, and Molnar 1. (1976) Solvophobic interactions in liquid chromatography with nonpolar stationary phases. J. Chromat. 125, 129-156.

Karger B. L., Snyder L. R. and Harvath C. (1976) An Introduction to Separation Science. Wiley, New York.

Karger B. L., Gant J. R., Hartkopf A., Weiner P. H. (1976) Hydrophobic effects in reverse phase liquid chromatography. J. Chromat. 128, 65-78.

Karickhoff S. (1984) Organic pollutant sorption in aquatic systems. J. Hydraul. Div. Am. Soc. civ. Engrs 110, $707-735$

Karickhoff S. W. and Brown D. S. (1979) Determination of octanol/water distribution coefficients, water solubilities, and sediment/water partition coefficients for hydrophobic organic pollutants. EPA-600/4-79-032, Athens.

Karickhoff S. W., Brown D. S. and Scott T. A. (1979) Sorption of hydrophobic pollutants on natural sediments. Wat. Res. 13, 241-248.

Kringstad K. P., Sousa F. and Stromberg L. M. (1984) Evaluation of lipophilic properties of mutagens present in spent chlorination liquid from pulp bleach. Envir. Sci. Technol. 18, 200-203.

Krstulovic A. M., Colin H., Guichon G. (1982) Comparison methods used for the determination of the void volume in reverse phase liquid chromatography. Analyt. Chem. 54, $2438-2443$.

Leo A. and Hansch C. (1971) Linear free-energy relationships between partitioning solvent systems. $J$. org. Chem. 36, 1539-1543.

Leo A. and Hansch C. (1979) Substituent Constant for Correlation Analysis in Chemistry and Biology. Wiley, New York

Leo A. Hansch C. and Elkins D. (1971) Partition coefficients and their uses. Chem. Rev. 71, 6 .

Locke D. C. (1974) Selectivity in reverse phase liquid chromatography using bonded stationary phases. $J$. Chromat. Sci., 12, 433437.

Mackay D. (1982) Correlation of bioconcentration factors. Envir. Sci. Technol. 16, 274-277.

McAuliffe C. (1966) Solubility in water paraffin, cycloparaffin, olefin, acetylene, cycloolefin, and aromatic hydrocarbons. J. phys. Chem. 70, 1267-1275.

McCall J. M. (1975) Liquid-liquid partition coefficients by high performance liquid chromatography. J. med. Chem. 18, 549-552

McDuffie B. (1980) Estimation of octanol/water partition coefficients for organic pollutants using reverse phase high performance liquid chromatography. Chemosphere, $10,73-83$.

Means J. C., Wood S. G., Hasset J. I. and Banwart W. L. (1980) Sorption of polycyclic aromatic hydrocarbons by sediments and soils. Envir. Sci. Technol. 14, 1524-1528.

Miller M. M.. Wasik S. P., Huang G., Shiu W. and MacKay D. (1985) Relationship between octanol/water partition coefficient and aqueous solubility. Envir. Sci. Technol. 19, $522-529$

Neely W. B., Branson D. P. and Blau G. E. (1974) The use of partition coefficients to measure the bioconcentration factors of organic compounds in fish. Envir. Sci. Technol. 8, $1113-1115$

Nemethy G. and Scheraga H. A. (1962) Structure of water and hydrophobic bonding in proteins-II. Model for thermodynamic properties of aqueous solutions of hydrocarbons. J. chem. Phys. 36, 3401-3411.

Rapaport R. A. and Eisenreich S. (1984) Chromatographic determinations of octanol-water partition coefficients for 58 polychlorinated biphenyl cogeners. Envir. Sci. Technol 18, $163-168$

Rekker R. F. (1977) The Hydrophobic Fragment Constant. Its Derivatization and Application. A Means of Characterizing Membrane Systems. Elsevier, New York.

Renberg L., Sundstrom G. and Sundh-Nygard K. (1980) Partition coefficients of organic chemicals derived from reverse phase thin layer chromatography. Chemosphere 9 , 683-691.

Soczewinski E. and Wachtmeister C. A. (1962) The relationship between the composition of certain ternary two phase solvent systems and $R_{m}$ values. $J$. Chromat. 7 , $311-319$

Sutton C. and Calder J. A. (1974) Solubility of higher molecular weight $n$-paraffins in distilled water and seawater. Envir. Sci. Technol. 8, 654-657.

Tsonopoulos C. and Prausnitz J. M. (1971) Activity coefficients of aromatic solutes in dilute aqueous solution Ind Engng Chem. Fund. 10, 593-599.

Unger S. H., Cook J. R. and Hollenberg J. S. (1978) Simple procedure for determining octanol-aqueous partition coefficients, distribution and ionization coefficients with reverse phase high performance liquid chromatography J. Pharm. Sci. 67, 1364-1367.

Valvani S. C., Yalkowski S. H. and Amidon G. L. (1976) Solubility of nonelectrolytes in polar solvents--IV Refinements in molecular surface area computations. J. phys. Chem. 80, 829-831

Vieth G. D., Austin N. M., Morris R. T. (1979) A rapid method for estimating $\log P$ for organic chemicals. Wat Res. 13, $43-47$.

Voice T. C.. Rice C. P. and Weber W. J. Jr (1983) Effect of solids concentration on the sorptive partitioning of hydrophobic pollutants in aquatic systems. Envir. Sci. Technol. 17, 513-517

Voice T. C. and Weber W. J. J $J_{\Gamma}$ (1983) Sorption of hydrophobic compounds by sediments soils and suspended solids-I. Theory and background. Wat. Res. 17, 1433-1441.

Yalkowski S. H. and Valvani S. C. (1979) Solubilities and partitioning 2. Relationships between aqueous solubilities, partition coefficients, and molecular surface areas of rigid aromatic hydrocarbons. J. Chem. Engng Data 24, 127-129.

Yalkowski S. H. and Valvani S. C. (1980) Solubility and partitioning-I. Solubility of nonelectrolytes in water. J. Pharm. Sci. 69, 912 $\cdots 922$ 